\title{
Estudio del efecto de la relación de intensidades relativas entre la onda de referencia y la onda objeto en holografía digital
}

\author{
Raúl Castañeda ${ }^{1}$, Diego Hincapiép ${ }^{1,2}$, Sebastián Bedoya ${ }^{1}$, Jorge Herrera-Ramírez ${ }^{2}$, Jorge Garcia-Sucerquia ${ }^{1, *}$ \\ ${ }^{1}$ Universidad Nacional de Colombia-Sede Medellín, Escuela de Física, Colombia \\ ${ }^{2}$ Instituto Tecnológico Metropolitano (ITM), Medellín, Colombia
}

\begin{abstract}
Resumen
En este trabajo se realizó un estudio para determinar la importancia de la relación relativa de las intensidades de la onda de referencia y la onda objeto en holografía digital en términos del contraste de la imagen holográfica reconstruida. Para ello se llevaron a cabo diferentes registros holográficos variando la relación de intensidades de la onda de referencia respecto a la onda objeto. Además de las reconstrucciones numéricas de estos registros holográficos, se hizo un análisis sobre los perfiles de intensidad de estos hologramas antes y después de realizado el requerido proceso de filtrado espacial. Los resultados obtenidos permiten concluir que contrario al proceso análogo de la holografía óptica tradicional, en holografía digital no es necesario mantener un alto control de las relaciones de intensidad entre la onda de referencia y la onda objeto. En general, se puede afirmar que esta relación de intensidades es de baja influencia para la calidad de la reconstrucción holográfica digital siempre y cuando que en el registro se respeten las adecuadas condiciones de muestreo y no saturación de los píxeles.
\end{abstract}

Palabras clave: holografía digital, filtrado espacial, procesamiento de imágenes.

Study of the relative ratio of the intensities between the reference and the object waves in digital holography

\begin{abstract}
In this paper, we present a study on the effect of the relative ratio of the intensities of the reference and object waves in digital holography with respect to the quality of the numerical reconstructions of the digitally recorded holograms. To perform the study, we have recorded several holograms of the same sample varying the ratio of intensities between the reference and object waves. In addition to the numerical reconstructions of these holograms, we have carried out an analysis of the intensity profiles of the digitally recorded holograms before and after performing the needed process of spatial filtering. The results of the numerical reconstructions and the analysis of the intensity profiles allow us to conclude that on contrary to the analogous process of optical holography, in digital holography is not necessary to keep a high control in the intensity ratio between the reference and object waves. In general, it can be stated that this relation of intensities is of low importance, while it is assured the correct sampling of the hologram and the no saturation of pixels are guaranteed.
\end{abstract}

Key words: Digital holography, spatial filtering, image processing.

\section{Introducción}

A pesar de que el concepto de holografía digital, entendida como la reconstrucción numérica de hologramas digitalmente registrados, nació casi simultáneamente con la holografía óptica (Goodman and Lawrence, 1967; Demetrakopoulos and Mittra, 1974), se puede considerar que no fue sino hasta el trabajo de Schnars y Jüptner de 1994 (Schnars and Jüptner, 1994) cuando se dio inicio al camino de aplicaciones e implementaciones en holografía digital que desde entonces han tenido un crecimiento continuo. Fueron las limitaciones en la resolución de las cámaras digitales (producto espacio-ancho de banda) y en las capacidades de procesamiento de los sistemas de cómputo lo que produjo que inicialmente esta idea estuviera estancada. A partir del desarrollo en estos prerrequisitos tecnológicos, la holografía digital ha tomado fuerza y ya se puede considerar como una rama separada dentro de lo que es la moderna óptica digital. Así, la holografía digital ahora tiene influencia en múltiples campos como la microscopía (Jericho S.K., et al., 2006; Herrera-Ramírez, et al., 2007; Colomb, et al., 2010; Garcia-Sucerquia, et al., 2006), la metrología (M. H. Jericho, et al., 2012; Schnars and Jueptner, 2005; Hincapie, Herrera, and Garcia-Sucerquia, 2007, Jericho S.K., et al., 2006), la formación de imágenes 3-D (Frauel

\footnotetext{
*Correspondencia:

Jorge Garcia-Sucerquia, jigarcia@unal.edu.co

Recibido: 3 de julio de 2015

Aceptado: 23 de septiembre de 2015
} 
et al., 2006; Javidi, et al., 2005), en el procesamiento de información de materiales (Hasegawa, Hayasaki, and Nishida, 2006), entre otros (Restrepo and GarciaSucerquia, 2013; Teeranutranont and Yoshimori, 2013).

La holografía digital ha alcanzado su desarrollo actual debido en gran parte a su versatilidad comparativa con respecto a su contraparte óptica. La conjugación de los sistemas digitales tanto en el registro de los hologramas como en la reconstrucción permite la inclusión de procesos que de manera analógica serían de limitada o más difícil aplicabilidad. Un ejemplo claro es la posibilidad de la eliminación de inconvenientes clásicos de la holografía óptica como son la presencia simultánea del orden cero y la imagen gemela en la visualización de la imagen holográfica reconstruida. Los métodos (Herrera-Ramírez and GarciaSucerquia, 2007; Takeda, Ina, and Kobayashi, 1982; Garcia-Sucerquia, Herrera-Ramírez, and VelasquezPrieto, 2004; Cuche, Marquet, and Depeursinge, 2000) para la eliminación del orden cero de difracción y la imagen gemela regularmente implementan eficientemente procesos de la óptica analógica aprovechando las ventajas del procesamiento digital de imágenes, con la idea de obtener reconstrucciones finales de mayor calidad.

Se han realizado muchos estudios para mejorar el contraste y la resolución espacial de los holograma reconstruido numéricamente (Garcia-Sucerquia, Herrera-Ramírez, and Velásquez-Prieto, 2005; Thurman and Bratcher, 2015; Maycock, et al., 2007; Hincapie D.A., et al., 2015; Xue, et al., 2014; Jeong, 2008). Desde la perspectiva de la holografía convencional, el contraste del holograma reconstruido depende en gran medida de la relación entre las intensidades de los haces de referencia y objeto en el registro de los hologramas. Esta relación de intensidades determina la visibilidad del patrón de interferencia que es el holograma, y esta visibilidad a su vez, controla la eficiencia de difracción del holograma, es decir, el brillo y contraste de la imagen reconstruida (Hariharan, 1996). Teóricamente se debe buscar la mayor visibilidad posible del patrón de interferencia y ésta se consigue cuando la relación entre las intensidades referencia a objeto de campos uniformes es de 1:1 (Hariharan, 1996). Sin embargo, en la práctica el haz objeto regularmente presenta variaciones espaciales de amplitud altas lo que hace que en el registro del holograma sea necesario trabajar con relaciones mayores que 1:1. Es decir, el haz de referencia, en el que por lo regular se puede asegurar una buena uniformidad, debe tener una intensidad mayor que la luz proveniente de cualquier punto del objeto, para evitar efectos no lineales y disminuir la influencia del ruido de intermodulación (Hariharan, 1996; Jeong, 2008). Esto implica relaciones referencia a objeto que van entre 2:1 hasta 10:1 dependiendo del tipo de holograma a ser registrado (Vorobyov, 2003; Jeong, 2008). Así, nuestro interés en este trabajo es analizar la importancia de la relación de intensidades entre las ondas de referencia y objeto en holografía digital y determinar el papel que juega en la calidad de la imagen reconstruida numéricamente.

Este artículo está organizado como sigue. En la siguiente sección se presenta el marco teórico básico de la holografía digital. En la sección de desarrollo experimental se describe el sistema experimental y los elementos computacionales que se han usado para llevar a cabo nuestro estudio, mostrando esquemáticamente la metodología implementada desde el registro digital de los hologramas, pasando por su procesamiento intermedio, hasta la reconstrucción numérica final. En la sección de resultados y discusión se presentan las reconstrucciones bajo diferentes relaciones de intensidad del haz referencia a haz objeto y el análisis sobre los histogramas de los diferentes hologramas. Adicionalmente, allí mismo se presenta la discusión de estos resultados. Finalmente, se presentan las conclusiones más importantes de este trabajo.

\section{Marco teórico}

La holografía digital puede entenderse como un proceso de formación de imágenes en dos pasos. El primero consiste en el registro digital de un patrón de interferencia, generado por la superposición en amplitud de una onda objeto y una onda de referencia mediante el uso de una cámara digital tipo CCD o CMOS (Schnars and Juptner, 2002). La cámara digital se encarga de transmitir y almacenar en la memoria de una computadora las intensidades que genera el patrón de interferencia, como un conjunto de datos que pueden ser representados en una imagen digital.

Un holograma digital usualmente se genera mediante una configuración óptica que está conformada por una fuente de luz coherente (láser), elementos ópticos para dirigir los haces de luz y finalmente un dispositivo de registro de luz, cámara CCD o CMOS. El haz de luz coherente proveniente del láser es dividido en dos haces mediante un divisor de haz BS (Beam Splitter). Uno de los haces se encarga de iluminar el objeto que se desea registrar, éste se conoce como haz objeto. La reflexión de este haz sobre la superficie del objeto se dirige al medio de registro. La segunda onda es dirigida directamente hacia el medio de registro y es denominado haz de referencia. Estos dos haces de luz interfieren generando un patrón de interferencia, que es el denominado holograma. La intensidad de este patrón de interferencia u holograma, es lo que registra la cámara digital y está dado por:

$$
H(x, y)=|R(x, y)+O(x, y)|^{2},
$$

donde $R(x, y)$ y $O(x, y)$ hacen referencia a la amplitud compleja de la onda de referencia y la onda objeto, respectivamente. Si se expande esta expresión se obtiene:

$$
H(x, y)=O O^{*}+R R^{*}+O R^{*}+R O^{*},
$$

donde * denota el complejo conjugado. Los dos primeros términos de la ecuación (2) hacen referencia al orden cero 
de difracción. El tercer y cuarto término son los denominados órdenes +1 y -1 de difracción, que dan cuenta de la imagen real y la imagen virtual en holografía.

El proceso de reconstrucción numérica de un holograma registrado digitalmente se puede describir como la simulación de la difracción de la onda de referencia a través del holograma registrado. En general, este problema está correctamente descrito por la teoría escalar de difracción de la luz ya sea por las fórmulas de Fresnel-Kirchhoff o Rayleigh-Sommerfeld (Goodman, 2005; Picart, 2015). Aunque estas fórmulas surgen de supuestos diferentes, se puede llegar a una equivalencia entre ellas si se asumen condiciones de iluminación de la abertura, o en este caso del holograma, con una onda plana y de observación en un plano a una gran distancia comparada con el tamaño del holograma. Estas condiciones suelen ser comunes en la práctica en holografía digital de forma tal que la formula de difracción se escribe como:

$\Gamma(\xi, \eta, d)=\frac{i}{\lambda} \iint_{-\infty}^{\infty} H(x, y) R(x, y) \frac{\exp (-i k r)}{r} \cos (\Theta) d x d y$

donde $\Gamma(\xi, \eta, d)$ representa el campo complejo reconstruido a una distancia perpendicular $d$ del plano del holograma. $r=\sqrt{(x-\xi)^{2}+(y-\eta)^{2}+d^{2}}$ es la distancia entre un punto $(x, y)$ en el plano del holograma y un punto $(\xi, \eta)$ en el plano de reconstrucción. $\mathrm{H}(x, y)$ es el holograma registrado que es iluminado con una onda $\mathrm{R}(x, y)$ y $\Theta$ es el ángulo entre el vector $r$ y la normal al plano del holograma. La figura 1 representa esquemáticamente este proceso de difracción.

En la práctica existen diferentes maneras de implementar numéricamente la ecuación 3 (Schnars, et al., 2015; Picart, 2015). Una de las formas más frecuentes de implementarla es usando la aproximación de Fresnel que permite un fácil computo numérico del campo complejo reconstruido a través de transformadas rápidas de Fourier (Picart, 2015; Schnars, et al., 2015). La ecuación 4 es la versión discreta que implementa esta aproximación en particular (Picart, 2015):

$$
\begin{aligned}
\Gamma(d)=\frac{\exp (i k d)}{i \lambda d} & \exp \left(\frac{i k}{2 d} \Delta \xi^{2} p^{2}\right) \sum_{n=-N / 2}^{(N / 2)-1} H(n) \\
& \exp \left(\frac{i k}{2 d} \Delta x^{2} n^{2}\right) \exp \left(-\frac{i k}{d} \Delta x n \Delta \xi p\right)
\end{aligned}
$$

Por simplicidad la ecuación 4 está escrita en una dimensión. $(\Delta x, \Delta \xi)$ son los tamaños del pixel en el plano del sensor de imágenes (plano holograma) y en el plano de reconstrucción (plano imagen), respectivamente. $\Gamma(d)$ representa la amplitud compleja reconstruida en un plano a una distancia $d$ desde el plano del holograma. $k=2 \pi / \lambda$ es el número de onda con longitud de onda de iluminación $\lambda$. En el régimen paraxial de propagación, que puede considerarse común en holografía digital, esta aproximación es relativamente precisa.

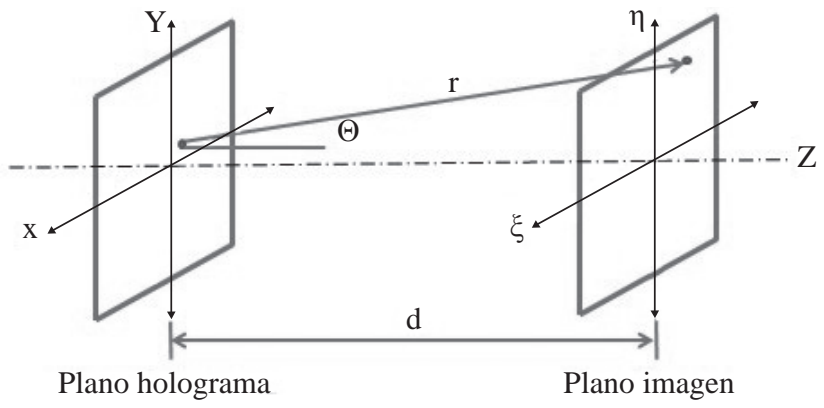

Figura 1. Diagrama de propagación en holografía digital.

\section{Desarrollo experimental}

Se llevó a cabo el registro de un conjunto de seis hologramas digitales, donde se cambió la relación de intensidad de la onda de referencia respecto a la intensidad de la onda objeto manteniendo la convención (X:Y) para denotar que mientras que la intensidad de la onda de referencia es $\mathrm{X}$ la de la onda objeto es Y. Se registraron hologramas con las relaciones (1:2), (1:1), (2:1), (3:1), (4:1) y (5:1) Se varió dicha relación desde (1:2) hasta (5:1).

Los hologramas registrados fueron realizados utilizando una configuración fuera de eje, mediante un montaje experimental basado en un interferómetro del tipo Mach-Zehnder (Figura 2) usando como fuente de luz coherente un láser de He-Ne con longitud de onda de $632.8 \mathrm{~nm}$ y una potencia de $35 \mathrm{~mW}$. Como medio de registro se utilizó una cámara digital CMOS (THORLABS - DCC1545M) de 1280 X 1024 píxeles y tamaño de pixel $5 \mu \mathrm{m}$ x $5 \mu \mathrm{m}$. La distancia de registro, desde el objeto al plano del sensor de la cámara, fue de $0.65 \mathrm{~m}$.

A partir de los hologramas registrados se llevó a cabo su reconstrucción numérica. Este proceso de reconstrucción fue realizado utilizando el plugin Numerical Propagation el cual es funcional en el software especializado para analizar y procesar imágenes digitales ImageJ (Piedrahita-Quintero, n.d.). Numerical propagation implementa diferentes estrategias numéricas para la realización de la propagación numérica de campos ópticos, entre las que se encuentra la transformada de Fresnel presentada anteriormente en la ecuación 4. Este software requiere como parámetros de reconstrucción las siguientes especificaciones del montaje experimental: Longitud de onda (Wavelength) $=632.8 \mathrm{~nm}$; la distancia de propagación (Distance) $=0.65 \mathrm{~m}$; el ancho del holograma (Input width) $=6.6 \mathrm{~mm}(1280 \times 5 \mu \mathrm{m})$ y la altura del holograma (Input Height) $5.4 \mathrm{~mm}(1024$ x $5 \mu \mathrm{m})$.

Ya que en el registro de estos hologramas fuera de eje se aseguró que existía una separación espacial adecuada de los diferentes órdenes de difracción, en la etapa de reconstrucción (Picart and Leval, 2008), se pudo llevar a cabo un proceso de filtrado espacial digital. Este filtrado permite eliminar el orden cero y la imagen gemela 


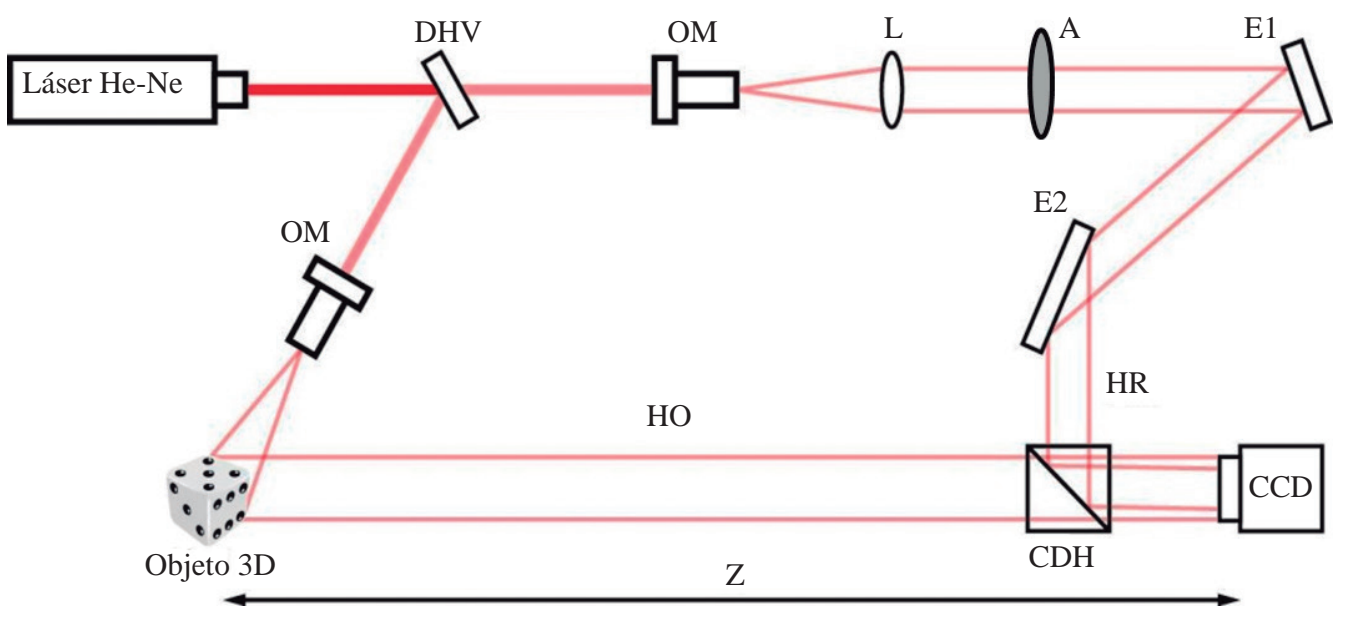

Figura 2. Montaje experimental. DHV: divisor de haz variable, OM: objetivo de microscopio, L: lente, A: atenuador, E1, E2: espejos, HO: haz objeto, HR: haz de referencia, CDH: cubo divisor de haz, CCD: cámara digital.

siguiendo el método propuesto en holografía digital por Cuche et al. (Cuche, Marquet, and Depeursinge, 2000) y desarrollado inicialmente por Takeda et al. (Takeda, Ina, and Kobayashi, 1982), donde se filtran las frecuencias asociadas al orden cero y la imagen gemela en la transformada de Fourier del holograma. De esta manera se mejora la calidad en la visualización de las imágenes reconstruidas, al realzar el contraste y reducir el ruido agregado por la imagen gemela y el orden cero de difracción. La figura 3 muestra esquemáticamente el resultado del proceso de reconstrucción de los hologramas sin realizar el proceso de filtrado espacial y usando el filtrado espacial.

La figura 4 es un diagrama de bloques de los procedimientos implementados que incluye los procesos de reconstrucción con filtrado y sin filtrado, y la extracción de perfiles de intensidades en los hologramas para su análisis posterior.

\section{Resultados y discusión}

La figura 5 muestra los resultados obtenidos en la reconstrucción de hologramas para dos objetos utilizados en este estudio, un dado y un modelo de un caballo. Para la obtención de estos resultados se aplicó el proceso descrito de filtrado espacial del holograma mediante transformada de Fourier para eliminar el orden cero y la imagen gemela.

En la figura 6 se presentan los resultados obtenidos para la reconstrucción de los diferentes hologramas registrados según la relación de intensidad usando como objeto un dado. La primera columna enseña los hologramas registrados, allí se hace evidente el incremento en el valor de la relación de los haces por el brillo perceptible en estas imágenes. La segunda columna contiene sus correspondientes transformadas de Fourier. La tercera columna de la figura 6, que corresponde a los resultados de reconstrucción del holograma sin usar filtrado espacial; se puede asemejar a lo que serían los resultados de

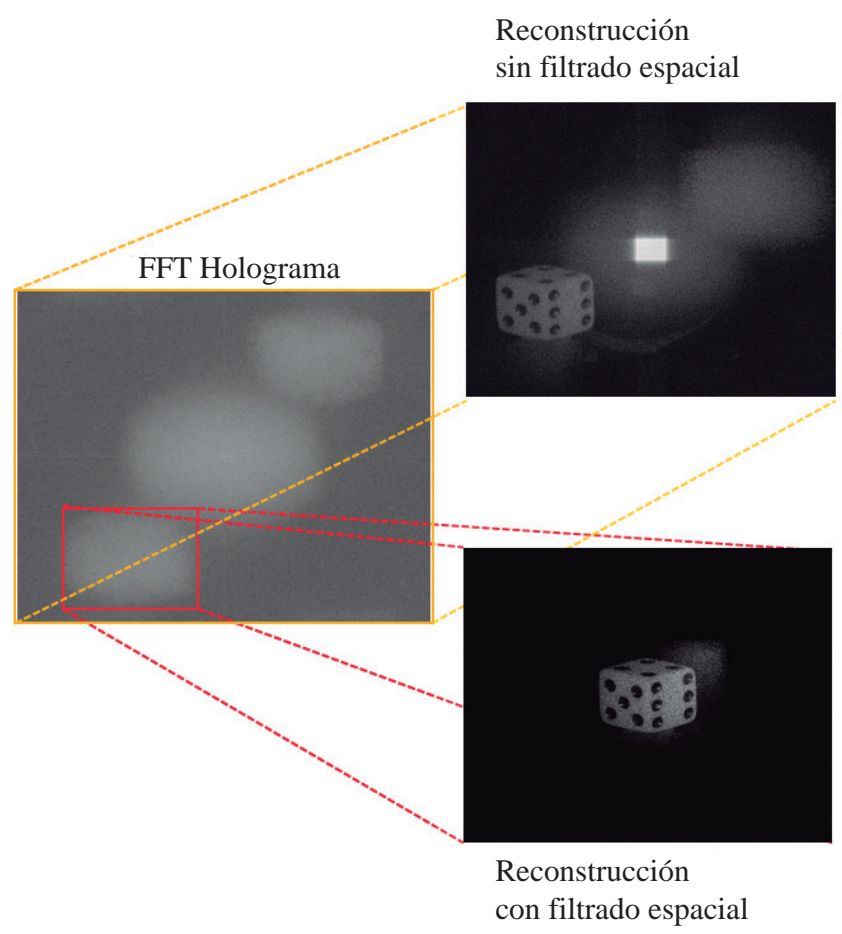

Figura 3. Proceso de reconstrucción del holograma. La línea amarilla indica el resultado de reconstrucción directa sin filtrado espacial. La línea roja indica el resultado de reconstruir el holograma utilizando el filtrado espacial, donde se consideran sólo las frecuencias espaciales de interés, orden +1 .

reconstrucción de los hologramas equivalentes obtenidos en holografía analógica. Como se puede observar en esta columna, la calidad de la imagen de interés en cuanto al brillo y contraste se ve afectada por la presencia del orden cero y la imagen gemela, entre otros ruidos de reflejos indeseables. En el caso de la reconstrucción análoga de hologramas, la eficiencia de difracción del holograma está 


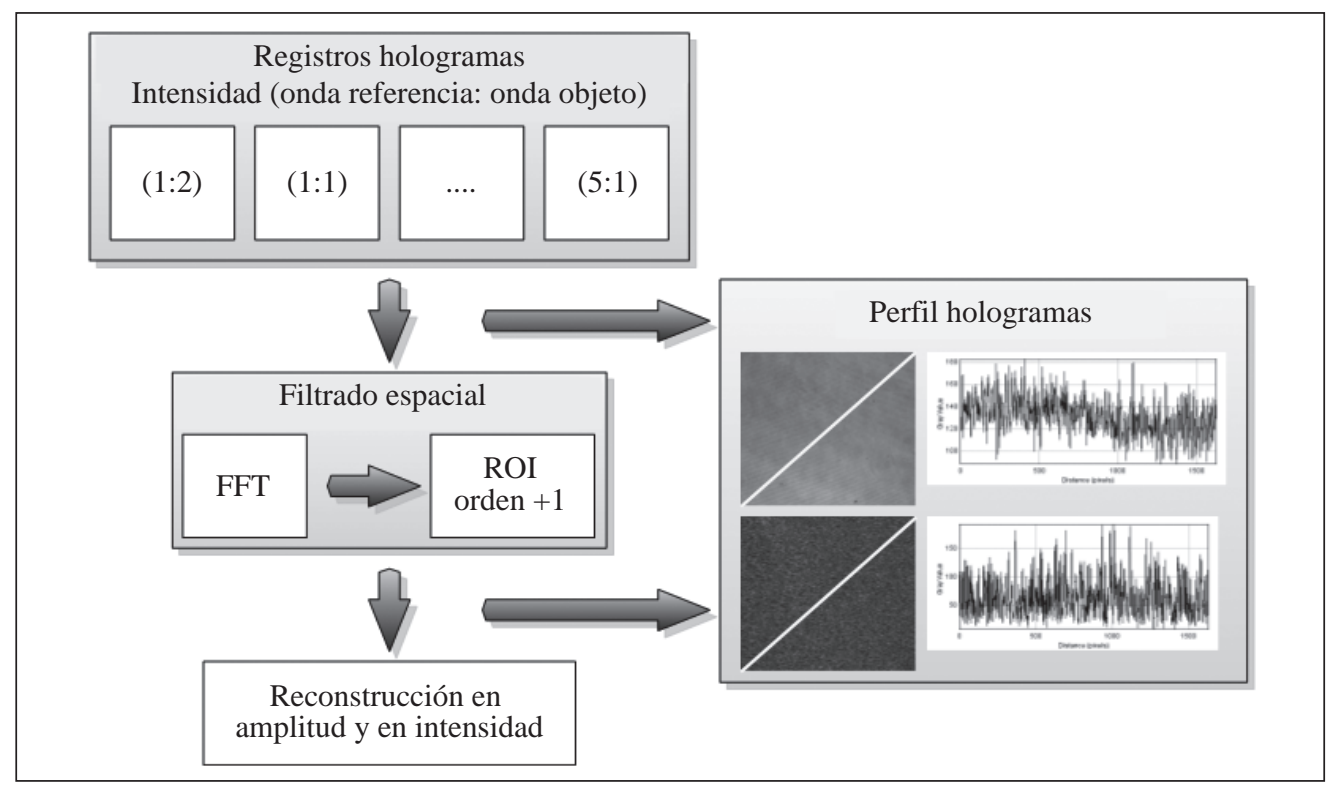

Figura 4. Diagrama de bloques de los procedimientos realizados desde el registro de los hologramas hasta la reconstrucción de estos mismos. Se incluyen también los procesos de filtrado espacial y de análisis comparativo de perfiles de intensidades entre los hologramas originales y los hologramas filtrados espacialmente.
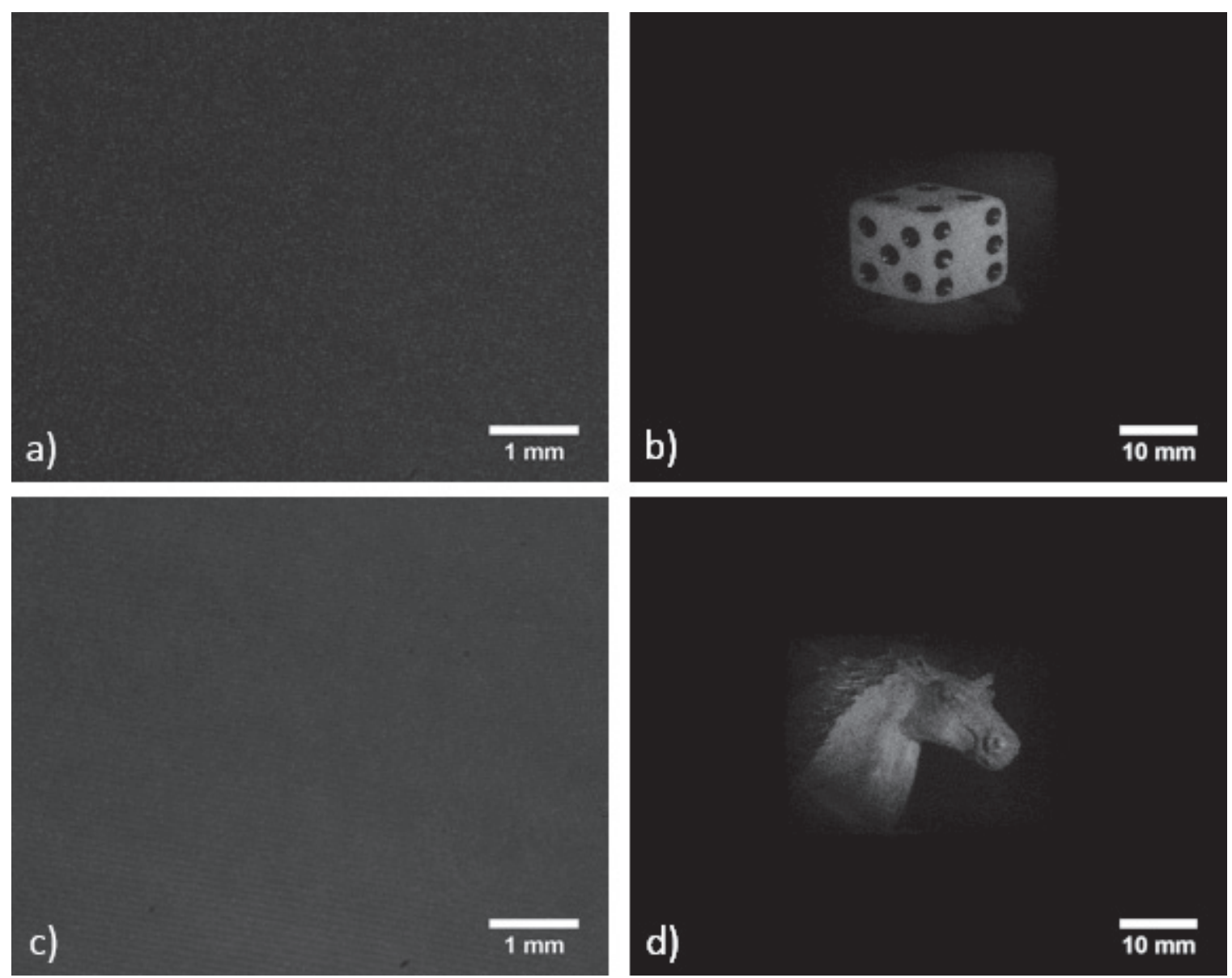

Figura 5. Imágenes de los hologramas registrado y reconstruidos. a) Holograma registrado para el dado, b) resultado de reconstrucción holográfica del dado, c) holograma registrado para el caballo, d) resultado de reconstrucción holográfica del caballo. 


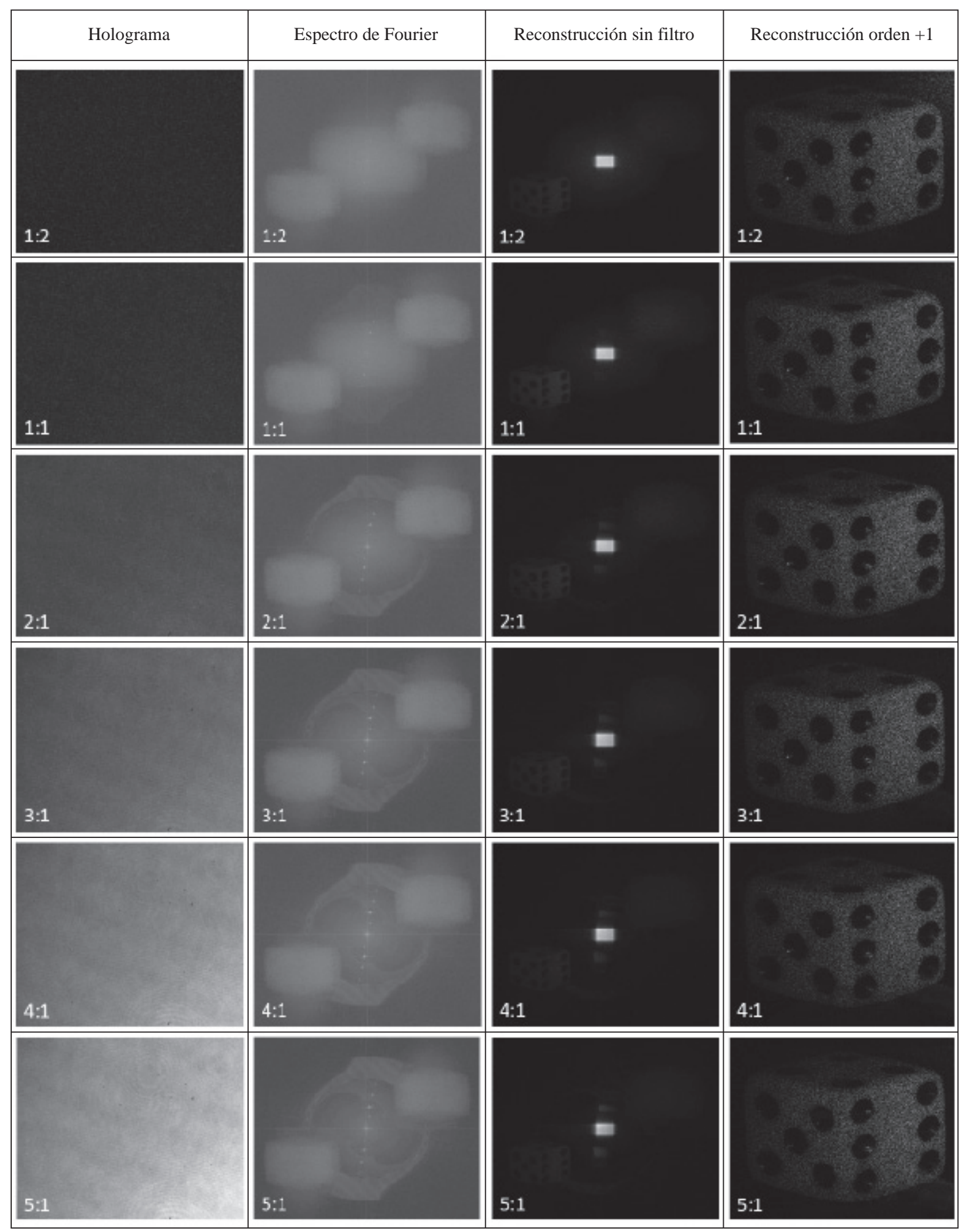

Figura 6. Resultados experimentales para el holograma de un dado cuando se cambia la relación de intensidades entre los haces de referencia y objeto. Columna 1: hologramas registrados. Columna 2: transformadas de Fourier. Columna 3: resultados de reconstrucción digital sin filtrado espacial. Columna 4: resultados de reconstrucción usando filtrado espacial.

determinada en gran parte por la relación de los haces de referencia y objeto, y por tanto, el cuidado de esta relación a la hora del registro determina la energía contenida en el orden cero y las imágenes gemelas en la reconstrucción. Adicional al requerimiento de buena eficiencia de difracción, la relación de intensidades también está ligada a la generación del ruido de intermodulación (Hariharan, 1996; Jeong, 2008), por lo que hay un compromiso para la elección de la relación de intensidades entre altas o bajas en el que no sólo se puede atender a la eficiencia 
de difracción del holograma como factor para su elección. La cuarta columna de la figura 6 muestra los resultados de reconstrucción de los hologramas al usar filtrado espacial. En estas imágenes no se observan diferencias notables en la calidad. En el caso de la holografía digital, gracias a sus ventajas para la manipulación de la información, el efecto negativo del orden cero, la imagen gemela y otros ruidos generalmente se pueden eliminar en su totalidad. Según esto, la relación de intensidades no es un factor de valor diferencial en la calidad de la imagen final reconstruida.

Con el fin de validar las observaciones anteriores, se hizo una réplica del experimento, utilizando un objeto de características diferentes al dado, un caballo de madera. La figura 7 muestra los resultados de la reconstrucción obtenida luego de realizar el proceso de filtrado espacial. Como se puede apreciar al igual que en el caso del dado, no hay cambios considerables en la calidad de las imágenes reconstruidas que puedan asociarse a la diferencia de intensidades entre los haces en el proceso de registro. Esta observación puede soportarse además con los resultados presentes en la figura 8. En la figura 8a. se muestran los valores promedio de la intensidad de los perfiles extraídos de los hologramas originales y los hologramas filtrados del dado, para las diferentes relaciones de intensidades entre haz de referencia y objeto. De la misma manera lo hace la figura 8b. para el modelo del caballo.

En esta figura 8 se puede observar que los promedios de intensidad en los hologramas filtrados son muy semejantes, indicando que la información del objeto está presente en todos los hologramas y que al usar el filtrado espacial se extrae esta información excluyentemente. Por tanto, la intensidad restante en los hologramas originales es la información que se puede interpretar como el ruido. En el caso de holografía análoga esta es la parte de la información que implica la redistribución de la energía en los otros órdenes de difracción que no corresponden a la imagen deseada. Es decir, se disminuye la eficiencia de la difracción, como lo ilustra la tercera columna de la figura 6. En el caso de la holografía digital este efecto se puede evitar y es así que la calidad de las reconstrucciones mostradas en la cuarta columna de la figura 6 y en la figura 7, evidenciadas en el brillo promedio y el contraste, son mejores permitiendo observar mejor los detalles y puntos brillantes de los objetos reconstruidos sin una influencia evidente de la relación de intensidades de los haces de referencia y objeto.

\section{Conclusiones}

Los resultados obtenidos muestran que la condición de relación de intensidad del haz de referencia y el haz objeto, con la que se debe ser cuidadoso en el registro de holografía análoga, no es una condición necesaria en la holografía digital para obtener un holograma reconstruido numéricamente con buena calidad, siempre y cuando se realice un proceso de filtrado espacial.

El análisis de los perfiles de intensidad de los hologramas registrados muestran que la relación de intensidad entre los haces del registro solo cambian el nivel promedio de dichos perfiles mientras que la información de interés del objeto, extraída mediante el proceso de filtrado espacial, se mantiene similar en todos ellos. Esto implica en cuanto a la información contenida en el holograma, que parte de ésta en el momento de la reconstrucción hace que la energía aportada por la iluminación de reconstrucción se redistribuya de manera ineficiente en los órdenes de difracción no deseados. Este efecto es anulado con el filtrado espacial antes de la reconstrucción.
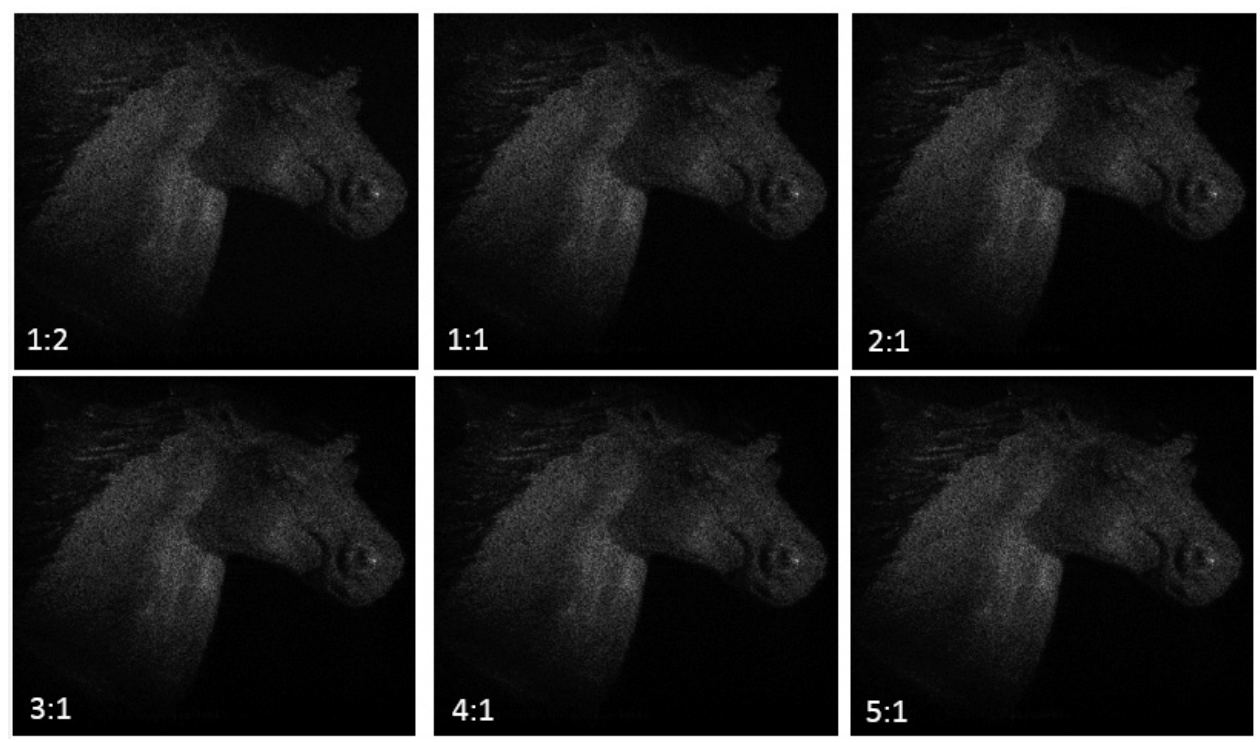

Figura 7. Resultados experimentales de reconstrucción para el holograma de un modelo de un caballo. Los resultados corresponden a las diferentes relaciones de intensidades usadas en este trabajo y usando filtrado espacial sobre los hologramas. 

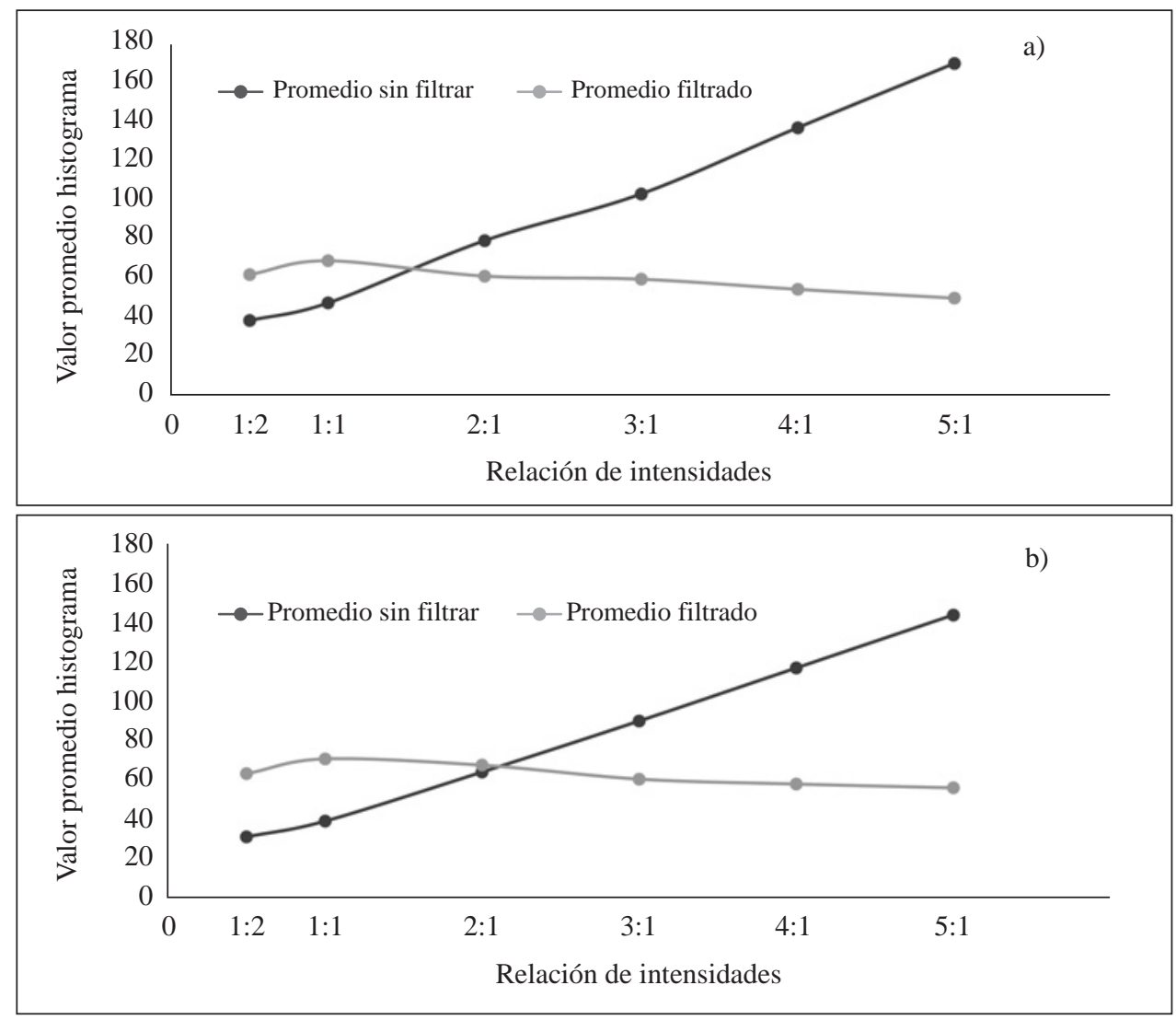

Figura 8. Promedio perfiles de intensidad: a) Valores promedio de las relaciones de intensidades de los hologramas del dado. b) Valores promedio de las relaciones de intensidad de los hologramas del caballo.

Lo anterior pone de manifiesto la ventaja de la holografía digital de poder manipular el holograma para extraer la información deseada, permitiendo que el cuidado estricto de la relación de intensidades de los haces de referencia y objeto durante el registro del holograma no sea un condicionante de alto valor para la calidad de las reconstrucciones holográficas numéricamente realizadas.

\section{Agradecimientos}

Los autores agradecen el soporte de la Universidad Nacional de Colombia, Proyecto Hermes 19384, el Programa de Internacionalización del Conocimiento y el programa de Jóvenes Investigadores de Colciencias-Universidad Nacional de Colombia código Hermes 28751. Adicionalmente este trabajo se realizó con apoyo parcial del proyecto financiado por el Instituto Tecnológico Metropolitano - ITM P14217.

\section{Conflicto de intereses}

Los autores declaran que no tienen conflicto de intereses.

\section{Referencias}

Colomb, T., Pavillon, N., Kühn, J., Cuche, E., Depeursinge, C., and Emery, Y. (2010). Extended Depth-of-Focus by Digital Holographic Microscopy. Opt. Lett., 35 (11). OSA, 1840-42.
Cuche, E., Marquet, P., and Depeursinge, C. (2000). Spatial Filtering for Zero-Order and Twin-Image Elimination in Digital off-Axis Holography. Applied Optics, 39 (23): 4070-75.

Demetrakopoulos, T., and Mittra, R. (1974). Digital and Optical Reconstruction of Images from Suboptical Diffraction Patterns. Applied Optics, 13 (3). Optical Society of America, 665-70.

Frauel, Y., Naughton, T., Matoba, O., Tajahuerce, E., and Javidi, B. (2006). Three-Dimensional Imaging and Processing Using Computational Holographic Imaging. Proceedings of the IEEE, 94 (3): 636-53.

Garcia-Sucerquia, J., Herrera-Ramírez, J., and VelasquezPrieto, D. (2004). DC Term Filtering Techniques in Digital Holography. Edited by Aristides Marcano O. and Jose Luis Paz. Proc. SPIE, 5622 (October): 1353-58.

Garcia-sucerquia, J., Herrera-Ramírez, J., and VelásquezPrieto, D. (2005). Improvement of the Signal-to-Noise Ratio in Digital Holography. Revista Mexicana de Física, 51 (1): 76-81.

Garcia-Sucerquia, J., Xu, W., Jericho, S., Klages, P., Jericho, M., and Kreuzer, H. (2006). Digital in-Line Holographic Microscopy. Appl. Opt., 45 (5). OSA, 836-50.

Goodman, J W, and Lawrence, R. (1967). Digital image formation from electronically detected holograms. Applied Physics Letters, 11 (3). 
Goodman, J. (2005). Introduction to Fourier Optics. Greenwood Village: Roberst \& Company Publishers.

Hariharan, P. (1996). Optical Holography: Principles, Techniques, and Applications. 2nd ed. Cambridge, UK: Cambridge University Press.

Hasegawa, S., Hayasaki, Y., and Nishida. N. (2006). Holographic Femtosecond Laser Processing with Multiplexed Phase Fresnel Lenses. Optics Letters, 31 (11). Optical Society of America, 1705.

Herrera-Ramírez, J., and Garcia-Sucerquia, J. (September, 2007). Digital off-Axis Holography without ZeroOrder Diffraction via Phase Manipulation. Optics Communications, 277 (2): 259-63.

Herrera-Ramírez, J., Mendoza, E., García,C., and Castaneda, R. (2007). Aplicación de La Microscopía Holográfica Digital En Transmisión Para La Caracterización Del Espesor de Recubrimientos Delgados. Scientia E Technica, 4 (36): 467-72.

Hincapie, D., Herrera, J., and Garcia-Sucerquia, J. (2007). Filtering of Phase-Difference Images in Digital Holography. Adaptive Optics: Analysis and Methods/Computational Optical Sensing and Imaging/Information Photonics/ Signal Recovery and Synthesis Topical Meetings on CDROM, June. Washington, D.C.: OSA, DTuD7.

Hincapie, D., Herrera-Ramirez J., and Garcia-Sucerquia, J. (2015). Single-Shot Speckle Reduction in Numerical Reconstruction of Digitally Recorded Holograms. Optics Letters, 40 (8).

Javidi, B., Ferraro, P., Seung-Hyun., De Nicola,S., Finizio,A., Alfieri,D., and Pierattini, G. (2005). Three-Dimensional Image Fusion by Use of Multiwavelength Digital Holography. Optics Letters, 30 (2). Optical Society of America, 144.

Jeong, T. (2008). Basic Principles and Applications of Holography BT - Fundamentals of Photonics. In Fundamentals of Photonics, 381-417.

Jericho, M., Kreuzer, J., Kanka, M., and Riesenberg, R. (2012). Quantitative Phase and Refractive Index Measurements with Point-Source Digital in-Line Holographic Microscopy. Appl. Opt., 51 (10). OSA, 1503-15.

Jericho, S., Garcia-Sucerquia, J., Xu, W., Jericho, M., and Kreuzer, J. (2006). Submersible Digital in-Line Holographic Microscope. Review of Scientific Instruments, 77 (4). AIP, 43706-10.

Maycock, J., Hennelly, B., McDonald, J., Frauel,Y., Castro, A., Javidi, B., and Naughton, T. (2007). Reduction of Speckle in Digital Holography by Discrete Fourier Filtering. J. Opt. Soc. Am. A, 24 (6). OSA, 1617-22.

Picart, P. (2015). New Techniques in Digital Holography. Edited by Pascal Picart. ISTE Ltd and John Wiley \& Sons, Inc.

Picart, P., and Leval, J. (2008). General Theoretical Formulation of Image Formation in Digital Fresnel Holography: Erratum. J. Opt. Soc. Am. A, 26 (2). OSA, 244.

Piedrahita, P., Castañeda, R., and Garcia-Sucerquia, J. (2015). Numerical wave propagation in ImageJ, App. Opt 54 (21): 6410-6415.

Restrepo, J., and Garcia-Sucerquia, J. (2013). Numerical Evaluation of the Limit of Concentration of Colloidal Samples for Their Study with Digital Lensless Holographic Microscopy. Appl. Opt., 52 (1). OSA, A310-18.

Schnars, U, and Jüptner, W. (1994). Direct Recording of Holograms by a CCD Target and Numerical Reconstruction. App. Opt., 33 (2), 179-81.

Schnars, U., Falldorf, C., Watson, J., and Jüptner, W. (2015). Digital Holography and Wavefront Sensing. 2nd ed. Springer Berlin Heidelberg.

Schnars, U., and Jueptner, W. (2005). Digital Holography: Digital Hologram Recording, Numerical Reconstruction, and Related Techniques. Digital Holography: Digital Hologram Recording, Numerical Reconstruction, and Related Techniques. Springer Berlin Heidelberg.

Schnars, U., and Juptner, W. (2002). Digital Recording and Numerical. Institute of Physics Publishing, 13: 17.

Takeda, M., Ina, H., and Kobayashi, S. (1982). Fourier-Transform Method of Fringe-Pattern Analysis for Computer-Based Topography and Interferometry. J. Opt. Soc. Am., 72 (1). OSA, 156-60.

Teeranutranont, S., and Yoshimori, K. (2013). Digital Holographic Three-Dimensional Imaging Spectrometry. App. Opt, 52 (1). Optical Society of America, A388-96.

Thurman, S., and Bratcher, A. (2015). Multiplexed SyntheticAperture Digital Holography. Applied Optics, 54 (3). OSA, 559.

Vorobyov, S. (2003). 24. Adjustment of Beams Ratio and Determination of Hologram's Exposure Time. 25 Holography Lessons,.

Xue, G., Liu, J., Li, X., Jia, J., Zhang, Z., Hu, B., and Wang, Y. (2014). Multiplexing Encoding Method for Full-Color Dynamic 3D Holographic Display. Optics Express, 22 (15). OSA, 18473. 